\title{
Magnetotransport in p-type Ge quantum well narrow wire arrays
}

\author{
P. J. Newton ${ }^{1 *}$, J. Llandro ${ }^{1}$, R. Mansell ${ }^{1}$, S. N. Holmes ${ }^{2}$, C. Morrison ${ }^{3}$, \\ J. Foronda ${ }^{3}$, M. Myronov ${ }^{3}$, D. R. Leadley ${ }^{3}$ and C. H. W. Barnes ${ }^{1}$ \\ ${ }^{1}$ Department of Physics, Cavendish Laboratory, University of Cambridge, J. J. \\ Thomson Avenue, Cambridge, CB3 OHE, United Kingdom \\ ${ }^{2}$ Toshiba Research Europe Limited, Cambridge Research Laboratory, 208 Cambridge \\ Science Park, Milton Road, CB4 OGZ, United Kingdom \\ ${ }^{3}$ Department of Physics, University of Warwick, Coventry, CV4 7AL, United Kingdom
}

\begin{abstract}
We report magnetotransport measurements of a SiGe heterostructure containing a 20 $\mathrm{nm}$ p-Ge quantum well with a mobility of $800,000 \mathrm{~cm}^{2} \mathrm{~V}^{-1} \mathrm{~s}^{-1}$. By dry etching arrays of wires with widths between $1.0 \mu \mathrm{m}$ and $3.0 \mu \mathrm{m}$ we were able to measure the lateral depletion thickness, built-in potential and the phase coherence length of the quantum well. Fourier analysis does not show any Rashba related spin-splitting despite clearly defined Shubnikov-de Haas oscillations being observed up to a filling factor of $v=22$. Exchange-enhanced spin-splitting is observed for filling factors below $v=9$. An analysis of boundary scattering effects indicates lateral depletion of the hole gas by $0.5 \pm 0.1 \mu \mathrm{m}$ from the etched germanium surface. The built-in potential is found to be $0.25 \pm 0.04 \mathrm{eV}$, presenting an energy barrier for lateral transport greater than the hole confinement energy. A large phase coherence length of $3.5 \pm 0.5 \mu \mathrm{m}$ is obtained in these wires at $1.7 \mathrm{~K}$.
\end{abstract}


*Corresponding author. Electronic mail: pjn32@cam.ac.uk

Investigations of spin transport in germanium have shown that it outperforms other material choices for future spintronic technologies. ${ }^{1-8}$ In the more extensively studied III-V semiconductors, the spin relaxation time is restricted by the hyperfine interaction $^{9}$ and spin-orbit coupling, ${ }^{10}$ leading to inherent limitations for these materials. In Ge these limitations are overcome, owing to the zero nuclear spin of its most abundant isotopes and the inversion symmetry of the Ge crystal suppressing the Dyakonov-Perel spin relaxation mechanism. ${ }^{11-13}$ In addition, hole transport in Ge has the added advantage that hole wavefunctions vanish at the nucleus, further supressing the hyperfine interaction. ${ }^{14}$ For the production of high-quality high-electron mobility transistors (HEMTs) and non-local spin injection and detection devices, SiGe heterostructures are also expected to offer advantages over bulk $\mathrm{Si}$ and Ge epilayers, owing to increased mobility ${ }^{12,15,16}$ and quantum confinement effects. ${ }^{2}$ Ideally spintronic devices would use SiGe heterostructures containing p-Ge quantum wells to fully utilise all of these advantages. However, many high-mobility heterostructures have shown significant parallel conduction through the SiGe buffer layers and the boron doped supply layer, masking the transport through the p-Ge quantum well. ${ }^{17,18}$ In order to produce high-quality spintronic devices, controlled tunnel injection and a method of controlling spin in the quantum well channel, such as the Rashba effect, ${ }^{18,19}$ are required. ${ }^{2,5}$ Knowledge of parameters such as the interfacial injection barrier thickness and phase coherence length are also necessary for the design of spintronic devices.

In this Letter we dry etch arrays of narrow wires containing a single p-Ge quantum well to determine the depletion thickness, built-in potential and phase coherence 
length of the quantum well. We also solve the problem of parallel conduction and observe the spin-split Shubnikov-de Haas effect in such heterostructures at $1.7 \mathrm{~K}$. We show that the spin-splitting is of exchange-enhanced $Z_{\text {eeman }}{ }^{20}$ in origin and find no evidence for the Rashba effect in this heterostructure.

A schematic diagram of the structure used in this work is shown in Fig. 1. Hall bars (with $75 \mu \mathrm{m}$ width) showed a mobility of $\mu=0.79 \times 10^{6} \mathrm{~cm}^{2} \mathrm{~V}^{-1} \mathrm{~s}^{-1}$ and a p-type carrier density of $p=2.1 \times 10^{11} \mathrm{~cm}^{-2}$ in the dark and $\mu=0.80 \times 10^{6} \mathrm{~cm}^{2} \mathrm{~V}^{-1} \mathrm{~s}^{-1}$ with $p=2.3 \times 10^{11}$ $\mathrm{cm}^{-2}$ in the dark after illumination (DAL). These devices displayed significant parallel conduction.

To eliminate this parallel conduction, optical lithography was used to define wires with widths between 1.0 and $3.0 \mu \mathrm{m}$ which were dry etched through the quantum well, to a depth of $0.6 \mu \mathrm{m}$, with $\mathrm{CF}_{4}$ plasma. This etch depth is sufficient to electrically isolate the wires at low temperature. Aluminium was thermally evaporated and annealed for eight minutes at a temperature of $250{ }^{\circ} \mathrm{C}$ in order to form ohmic contacts to the p-Ge quantum well. Ohmic behaviour was observed down to the lowest measurement temperature of $1.7 \mathrm{~K}$. The wires were $1000 \mu \mathrm{m}$ long with effective conducting widths varying from 0.3 to $1.8 \mu \mathrm{m}$. Arrays of between 60 and 100 wires were connected at the $\mathrm{Al}$ ohmic contacts and measured in parallel. This prevented the magnetoresistance signal being dominated by mesoscopic conductance fluctuations ${ }^{21}$ or by intrinsic or processing-related defects.

Magnetotransport measurements were carried out in a perpendicular magnetic field up to $8 \mathrm{~T}$ at $1.7 \mathrm{~K}$ with either ac currents of $\sim 1 \mathrm{nA}$ per wire or dc currents in an ac modulation field of $6 \mathrm{mT}$ at $33 \mathrm{~Hz}^{22}$ Analogue $\mathrm{d} R_{\mathrm{xx}} / \mathrm{d} B$ and $\mathrm{d}^{2} R_{\mathrm{xx}} / \mathrm{d} B^{2}$ signals were measured using field modulation, enhancing the signal-to-noise ratio over the numerical derivative by a factor of approximately 100 . For DAL measurements, in- 
situ illumination was from a red light emitting diode close to the device. A persistent enhancement of the hole density was always observed after illumination.

In Fig. 2, the magnetoresistance is shown for the $2 \mu \mathrm{m}$ width wires in the dark (Fig. 2a) and DAL (Fig. 2b). The carrier density is $p=1.9 \times 10^{11} \mathrm{~cm}^{-2}$ in the dark and is $p=$ $2.1 \times 10^{11} \mathrm{~cm}^{-2}$ in DAL. The Landau level filling factors $(v)$ are indicated for the Shubnikov-de Haas minima to $v=10$, with spin-splitting at odd filling factors up to $v=5$. In wide Hall bar devices, parallel conduction through the supply layer and buffer layers distorts the quantum Hall plateaux in resistivity $\rho_{\mathrm{xy}}$, 'inverting' the $\rho_{\mathrm{xx}}$ resistivity minima in the Shubnikov-de Haas effect, ${ }^{17}$ however these effects are not seen in these narrow wire devices. There is a peak in the resistance at a field of $\sim 0.03$ T. This peak is not sharp enough to be due to weak anti-localization ${ }^{23}$ but can instead be attributed to a boundary scattering effect. ${ }^{24,25}$ This is observed in the non-depleted wires for all wire widths and can be used to estimate the conducting width ( $\left.W_{\text {eff }}\right)$ of the wire. If the boundary scattering field is at $B_{\mathrm{BS}}$, the effective wire width is given by equation (1) for a wire with hole density $p$,

$$
W_{e f f}=0.55 \frac{\hbar \sqrt{2 \pi p}}{e B_{B S}}
$$

These $2 \mu \mathrm{m}$ wires therefore have an effective wire width of $W_{\text {eff }}=1.1 \mu \mathrm{m}$.

Fig. 3a shows the analogue $-\mathrm{d}^{2} R_{\mathrm{xx}} / \mathrm{d} B^{2}$ signal of the wires with $W_{\text {eff }}=1.1 \mu \mathrm{m}$. This structure can be observed to a much higher filling factor; clear oscillations up to $v=$ 22 can be identified in this case. A plot of filling factor against inverse magnetic field $\left(1 / B_{\min }\right)$ is shown in Fig. $3 \mathrm{~b}$. The linear fit to this plot is $8.03 \times\left(1 / B_{\min }\right)$. At filling factor $v=11$, a spin-splitting is visible. The system is spin-split at filling factors less than $v=9$. These data show a strictly linear behaviour, since there is no onedimensional subband magnetic depopulation in these narrow wires. ${ }^{26}$ The number of 
one-dimensional subbands $N_{s}$ can be deduced from the value of $W_{\text {eff }}$ given by equation (1). We find $N_{s}=\hbar p / e B_{\mathrm{BS}} \approx 10$ in the wire where $\mathrm{W}_{\mathrm{eff}}=0.3 \mu \mathrm{m} .^{27}$

Fig. 4a shows the analogue $\mathrm{d} R_{\mathrm{xx}} / \mathrm{d} B$ signal in an array of only $25 \mathrm{~W}_{\text {eff }}=1.1 \mu \mathrm{m}$ wide wires. Reproducible conductance fluctuations are seen in the shaded area, up to $v=30$. The two traces are from field sweeps in opposite directions, confirming the reproducibility of this structure that is quasi-periodic in field. This structure was also observed when measuring arrays of more wires but was not observed in the (less sensitive) analogue $R_{\mathrm{xx}}$ signal.

The fast Fourier transform (FFT) of the low field $\mathrm{d} R_{\mathrm{xx}} / \mathrm{d} B$ signal is shown in Fig. $4 \mathrm{~b}$ in the magnetic field domain to $1.1 \mathrm{~T}$, avoiding the structure in the shaded area where conductance fluctuations can be seen. The main peak (at $\sim 4 \mathrm{~T}$ ) corresponds to the fundamental field $B_{\mathrm{f}}=h p / 2 e$, with spin-degeneracy $\left(g_{\mathrm{s}}\right)=2$ and a carrier density of $p$ $\sim 2 \times 10^{11} \mathrm{~cm}^{-2}$, which is the same as that obtained from the gradient of Fig. $3 \mathrm{~b}$. The peak at $\sim 8 \mathrm{~T}$ arises from spin-splitting. The peak close to zero frequency in the FFT is due to the background variation in $\mathrm{d} R_{\mathrm{xx}} / \mathrm{d} B$ (see Fig. 4a).

We do not observe a weak anti-localization peak close to zero applied field in any of the wire array structures. Beating in the amplitude of the Shubnikov-de Haas oscillations characteristic of spin-splitting caused by the Rashba effect is also not observed. There is no beating of the oscillatory structure in $\mathrm{dR}_{\mathrm{xx}} / \mathrm{dB}$ or FFT peak splitting in these wires (Fig. 4) that is characteristic of the Rashba effect. ${ }^{28}$ The presence of two regimes in plots of $v$ against $1 / \mathrm{B}_{\min }$ (see Fig. 3b), spin-split and nonspin-split, can be attributed to the onset of exchange-enhanced Zeeman splitting. This is characteristic of the magnetic field induced 'Stoner transition' seen in the GaAs two-dimensional system ${ }^{29}$ rather than a Rashba spin-orbit interaction. Observations of the cubic Rashba effect have been reported in samples with a carrier density of $p=$ 
$6 \times 10^{11} \mathrm{~cm}^{-2}$ and $p=1.5 \times 10^{12} \mathrm{~cm}^{-2} \cdot{ }^{18,19}$ At the lower carrier density here, Zeeman splitting dominates the low magnetic field spin-splitting. The compressive strain in the layers may be increasing the structural asymmetry in the band structure. This strain is certainly higher in the devices measured in Ref. 19 , owing to the $\mathrm{Si}_{0.5} \mathrm{Ge}_{0.5}$ barrier material providing a $2.1 \%$ strain in the p-Ge quantum well. In the wires measured in this work the Ge-rich $\mathrm{Si}_{0.2} \mathrm{Ge}_{0.8}$ barrier results in a reduced strain of $0.65 \%$ in the p-Ge quantum well. ${ }^{15}$ This reduced strain and lower carrier density could possibly minimise any Rashba effect. Further strain engineering in these SiGe heterostructures could therefore potentially lead to a controllable Rashba effect.

Fig. 5 shows the measured carrier density as a function of effective wire width in the dark and DAL. In the narrowest device measured, the wire is depleted in the dark and conduction is solely through the supply and buffer layers. Each wire has a very large impedance of $\sim 6 \mathrm{M} \Omega$. In DAL a carrier density of $p=2.2 \times 10^{11} \mathrm{~cm}^{-2}$ was measured. The boundary scattering peak ${ }^{24}$ occurred at a magnetic field of $0.16 \mathrm{~T}$ and indicates an effective wire width of $0.3 \mu \mathrm{m}$. The persistent conductivity in DAL is approximately independent of wire width. Secondary Ion Mass Spectroscopy measurements ${ }^{30}$ show that the background boron doping is less than $10^{17} \mathrm{~cm}^{-3}$ in the buffer layer. This freezes out at low temperature, leaving just the doping supply layer (see Fig. 1) as the source of charge. The parallel conduction measured in wide Hall bars is depleted in the case of each of the arrays of narrow wires in the dark and DAL. The carrier density in the wires before illumination is consistently lower than in the wide Hall bar devices for all wire widths, owing to edge depletion.

The depletion thickness $\left(x_{\mathrm{b}}\right)$ of the wires was estimated by comparing the effective wire width with the physical width determined by atomic force microscopy (AFM). The measured depletion thickness is between 0.37 and $0.62 \mu \mathrm{m}$ and is weakly 
dependent on the physical wire width. The median value of the calculated depletion thickness $\left(x_{\mathrm{b}}=0.49 \mu \mathrm{m}\right)$ at the dry etched Ge surface is shown for comparison in Fig. 5. The surface built-in potential $\left(e V_{\mathrm{bi}}\right)$ can estimated from Gauss's law using equation $(2)$,

$$
e V_{b i}=\frac{e p_{b} x_{b}^{2}}{2 \varepsilon_{0} \varepsilon_{r}}
$$

where $\varepsilon_{\mathrm{r}}=15.8$ is the relative permittivity of Ge. The charge density, $p_{\mathrm{b}}$, is the bulk equivalent of the measured sheet density $(p)$ in a wire of width $W_{\text {eff. }}$ In each of the wires the surface potential is $V_{\mathrm{bi}}=0.25 \pm 0.04 \mathrm{eV}$. In a spintronic device, this would represent the tunnel barrier for lateral spin injection from a ferromagnetic metal ${ }^{31}$ into the strained p-Ge quantum well at the etched Ge surface. The role of interface states in determining the dominance of spin accumulation over spin-injection is therefore an important consideration ${ }^{5}$ for design of spintronic devices in $\mathrm{p}-\mathrm{Ge}$ two-dimensional hole gases (2DHG).

The phase coherence length in the wires is calculated from the dephasing time, $\tau_{\phi}$, in a quasi-one-dimensional system ${ }^{32}$ with,

$$
\frac{1}{\tau_{\phi}}=\frac{\pi(k T)^{2}}{2 \hbar E_{F}} \ln \left(\frac{E_{F}}{k T}\right)+\left(\frac{k T}{\sqrt{D} W_{e f f} P_{0} \hbar^{2}}\right)^{\frac{2}{3}}
$$

where $T$ is the measurement temperature, $E_{\mathrm{F}}$ is the Fermi energy, $D$ is the diffusion constant and $P_{\mathrm{o}}$ is the density of states in the valence band of the Ge quantum well. The hole effective mass used to determine $P_{\mathrm{o}}$ has been measured to be $0.063 m_{\mathrm{e}}$ in similar Ge quantum well structures, ${ }^{33}$ where $m_{\mathrm{e}}$ is the free electron mass. Using equation (3), the phase coherence length is calculated to be $3.5 \pm 0.5 \mu \mathrm{m}$ at $1.7 \mathrm{~K}$ over the range of effective wire widths from $0.3 \mu \mathrm{m}$ to $1.8 \mu \mathrm{m}$. This phase coherence length is larger than typical quantum dot structures used for qubit manipulation ${ }^{34}$ and 
therefore offers possible applications for these $\mathrm{p}$-Ge 2DHGs in future quantum technologies.

In conclusion, we have etched arrays of $1.0 \mu \mathrm{m}$ to $3.0 \mu \mathrm{m}$ width wires, each containing single a single high mobility $\left(\mu=800,000 \mathrm{~cm}^{2} \mathrm{~V}^{-1} \mathrm{~s}^{-1}\right) \mathrm{p}-\mathrm{Ge}$ quantum well, and have removed the previously observed parallel conduction through buffer and supply layers. These samples have a carrier density of $p=2 \times 10^{11} \mathrm{~cm}^{-2}$ and we observe clear Shubnikov-de Haas oscillations up to a filling factor of $v=22$ at $1.7 \mathrm{~K}$. We do not observe any spin-splitting characteristic of the cubic Rashba effect recently seen in higher carrier density p-Ge and attribute spin-splitting of the Subnikov-de Haas oscillations up to $v=9$ to the exchange-enhanced Zeeman effect. In these wires, the lateral depletion thickness is $0.5 \pm 0.1 \mu \mathrm{m}$ at the dry etched Ge surface. The built-in potential is $0.25 \pm 0.04 \mathrm{eV}$, presenting an energy barrier for lateral spin-injection into a spintronic device that is greater than the hole confinement energy at the p-Ge surface. We calculated a large phase coherence length in the wires of $3.5 \pm 0.5 \mu \mathrm{m}$. The attainment of these parameters in p-Ge quantum wells, as well as the supressed hyperfine interaction, indicates that this material has applications in spintronic devices. Our results are consistent with the main source of spin-relaxation in the Ge wires being the Elliot-Yafet mechanism, ${ }^{35}$ where the spin-lifetime is longer for higher mobilities.

\section{Acknowledgements}

This work programme is funded by the EPSRC funded "Spintronic device physics in Si/Ge heterostructures" EP/J003263/1 and EP/J003638/1 projects and a platform grant, $\mathrm{EP} / \mathrm{J} 001074 / 1$. 


\section{REFERENCES}

${ }^{1}$ C. Shen, T. Trypiniotis, K. Y. Lee, S. N. Holmes, R. Mansell, M. Husain, V. Shah, H. Kurebayashi, I. Farrer, C. H. deGroot, D. R. Leadley, G. Bell, E. H. C. Parker, T. Whall, D. A. Ritchie and C. H. W. Barnes, Appl. Phys. Lett. 97, 162104 (2010)

${ }^{2}$ Y. Zhou, W. Han, L.-T. Chang, F. Xiu, M. Wang, M. Oehme, I. A. Fischer, J. Schulze, R. K. Kawakami, and K. L. Wang, Phys. Rev. B 84, 125323 (2011)

${ }^{3}$ Y. Hu, H. O. H. Churchill, D. J. Reilly, Jie Xiang, C. M. Lieber, and C. M. Marcus, Nature Nanotechnology 2, 622 (2007)

${ }^{4}$ H. Saito, S. Watanabe, Y. Mineno, S. Sharma, R. Jansen, S. Yuasa, K. Ando, Solid State Comm. 151, 1159 (2011)

${ }^{5}$ A. Jain, J.-C. Rojas-Sanchez, M. Cubukcu, J. Peiro, J. C. Le Breton, E. Prestat, C. Vergnaud, L. Louahadj, C. Portemont, C. Ducruet, V. Baltz, A. Barski, P. BayleGuillemaud, L. Vila, J.-P. Attané, E. Augendre, G. Desfonds, S. Gambarelli, H. Jaffrès, J.-M. George, and M. Jamet, Phys. Rev. Lett. 109, 106603 (2012)

${ }^{6}$ F. Pezzoli, F. Bottegoni, D. Trivedi, F. Ciccacci, A. Giorgioni, P. Li, S. Cecchi, E. Grilli, Y. Song, M. Guzzi, H. Dery, and G. Isella, Phys. Rev. Lett. 108, 156603 (2012)

${ }^{7}$ C. Payette, K. Wang, P. J. Koppinen, Y. Dovzhenko, J. C. Sturm, and J. R. Petta, Appl. Phys. Lett. 100, 043508 (2010)

${ }^{8}$ D. J. Paul, Semicond. Sci. Technol. 19, R75 (2004)

${ }^{9}$ J. R. Petta, A. C. Johnson, J. M. Taylor, E. A. Laird, A. Yacoby, M. D. Lukin, C. M. Marcus, M. P. Hanson, and A. C. Gossard, Science 309, 2180 (2005)

${ }^{10}$ M. Cardona, N. E. Christensen, and G. Fasol, Phys. Rev. B 38, 1806 (1988)

${ }^{11}$ E. Yablonovitch, H. W. Jiang, H. Kosaka, H. D. Robinson, D. S. Rao, and T. Szkopek, Proc IEEE 91, 761 (2003)

${ }^{12}$ E. J. Loren, J. Rioux, C. Lange, J. E. Sipe, H. M. van Driel, and A. L. Smirl Phys. Rev. B 84, 214307 (2011)

${ }^{13}$ C. Guite, V. Venkataraman, Appl. Phys. Lett. 101, 252404 (2010)

${ }^{14}$ M. H. Kolodrubetz, and J. R. Petta, Science 325, 42 (2009)

${ }^{15}$ A. Dobbie, M. Myronov, R. J. Morris, A. H. A. Hassan, M. J. Prest, V. A. Shah, E. H. C. Parker, T. E. Whall, and D. R. Leadley, Appl. Phys. Lett. 101, 172108 (2012)

${ }^{16}$ O.A. Mironov, A. H. A. Hassan, R. J. H. Morris, A. Dobbie, M. Uhlarz, D. Chrastina, J. P. Hague, S. Kiatgamolchai, R. Beanland, S. Gabani, I. B. Berkutov, M. Helm, O. Drachenko, M. Myronov, and D. R. Leadley, Thin Solid Films 557, 329 (2014) 
${ }^{17}$ C. Morrison, M. Myronov, J. Foronda, C. Casteleiro, J. E. Halpin, S. D. Rhead and D. R. Leadley, Silicon-Germanium Technology and Devices meeting $7^{\text {th }}$ ISTDM 105$106(2014)$

${ }^{18}$ C. Morrison, P. Wiśniewski, S. D. Rhead, J. Foronda, D. R. Leadley, and M. Myronov, App. Phys. Lett., 105, 182401 (2014)

${ }^{19}$ R. Moriya, K. Sawano, Y. Hoshi, S. Masubuchi, Y. Shiraki, A. Wild, C. Neumann, G. Abstreiter, D. Bougeard, T. Koga and T. Machida, Phys. Rev. Lett. 113, 086601 (2014)

${ }^{20}$ T. Ando, and Y. Uemura, J. Phys. Soc. Jpn. 37, 1044 (1974)

${ }^{21}$ C. P. Umbach, S. Washburn, R. B. Laibowitz, and R. A. Webb, Phys. Rev. B 30, 4048(R) (1984)

${ }^{22}$ D. Shoenberg, Magnetic Oscillations in Metals, (Cambridge University Press, Cambridge, 1984)

${ }^{23}$ B. Grbić, r. Leturcq, T. Ihn, K. Ensslin, D. Reuter, and A. D. Wiek, Phys. Rev. B 77, $125312(2008)$

${ }^{24}$ T. J. Thornton, M. L. Roukes, Axel Scherer and B. P. Van de Gaag, Phys. Rev. Lett. 63, 2128 (1989)

${ }^{25}$ E. Ditlefsen, and J. Lothe, Philosophic Magazine 14, 759 (1966)

${ }^{26}$ K. -F. Berggren, and D. J. Newson, Semicond. Sci. Technol. 1327 (1986)

${ }^{27}$ H. Akera, and T. Ando, Phys. Rev. B 43, 11676 (1991)

${ }^{28}$ G. Engels, J. Lange, T. Schäpers, and H. Lüth, Phys. Rev. B 55, R1958 (1997)

${ }^{29}$ B. A. Piot, D. K. Maude, M. Henini, Z. R. Wasilewski, K. J. Friedland, R. Hey, K. H. Ploog, A. I. Toropov, R. Airey and G. Hill, Phys. Rev. B 72, 245325 (2005)

${ }^{30}$ A. H. A. Hassan, O. A. Mironov, A. Dobbie, J. H. Morris, J. E. Halpin, V. A. Shah, M. Myronov, D. R. Leadley, IEEE 33 ISCEN Conference, 51 (2013)

${ }^{31}$ C. W. Burrows, A. Dobbie, M. Myronov, T. P. Hase, S. B. Wilkins, M. Walker, J. J. Mudd, I. Maskery, M. Lees, C. F. McConville, D. R. Leadley and G. R. Bell, Cryst. Growth Des. 13, 4923 (2013)

${ }^{32}$ K. K. Choi, D. C. Tsui, K. Alavi, Phys. Rev. B 36, 7751 (1987)

${ }^{33}$ O. A. Mironov, A. H. A. Hassan, M. Uhlarz, S. Kiatgamolchai, A. Dobbie, R. J. H. Morris, J. E. Halpin, S. D. Rhead, P. Allred, M. Myronov, S. Gabani, I. B. Berkutov, and D. R. Leadley, Phys. Status Solidi C11, 61 (2014) 
${ }^{34}$ J. R. Petta, A. C. Johnson, J. M. Taylor, E. A. Laird, A. Yacoby, M. D. Lukin, C. M. Marcus, M. P. Hanson, and A. C. Gossard, Science 3092180 (2005)

${ }^{35}$ I. Zutic, J. Fabian, S. Das Sarma, Rev. Mod. Phys. 76, 323 (2004) 


\section{FIGURE CAPTIONS}

Figure 1. A schematic diagram of the strained Ge quantum well structure. The Boron doping density is $2.4 \times 10^{18} \mathrm{~cm}^{-3}$ in the $20 \mathrm{~nm}$ thick doped region and $5 \times 10^{18} \mathrm{~cm}^{-3}$ in the $\delta$-doped region.

Figure 2. Shubnikov-deHaas effect in (a) the dark and (b) DAL in the $2 \mu \mathrm{m}$ wide wires where $W_{\text {eff }}=1.1 \mu \mathrm{m}$. Inset: the boundary scattering peak of these wires

Figure 3. (a) $-\mathrm{d}^{2} R_{\mathrm{xx}} / \mathrm{d} B^{2}$ of an array of wires where $W_{\text {eff }}=1.1 \mu \mathrm{m}$ at $p=1.9 \times 10^{11} \mathrm{~cm}^{-2}$ in the dark with filling factors indicated.(b) Filling factor against $1 / B_{\min }$ for the Shubnikov-de Haas minima positions in $R_{\mathrm{xx}}$.

Figure 4. (a) low magnetic field structure in $\mathrm{d} R_{\mathrm{xx}} / \mathrm{d} B$ from a wire array where $W_{\text {eff }}=$ 1.1 $\mu \mathrm{m}$. An increasing and a decreasing field sweep are shown for comparison. (b) an FFT from the magnetic field domain 0.2 to $1.1 \mathrm{~T}$.

Figure 5. The hole density from the Shubnikov-de Haas effect as a function of effective wire width in the dark and DAL. The average depletion thickness $\left(x_{\mathrm{b}}\right)$ is indicated. 


\section{FIGURES}

Figure 1

\begin{tabular}{|c|c|}
\hline Si cap & $2 \mathrm{~nm}$ \\
\hline $\mathrm{Si}_{0.2} \mathrm{Ge}_{0.8}$ & $30 \mathrm{~nm}$ \\
\hline B doped $\mathrm{Si}_{0.2} \mathrm{Ge}_{0.8}$ & $20 \mathrm{~nm}$ \\
\hline $\mathrm{Si}_{0.2} \mathrm{Ge}_{0.8}$ spacer & $20 \mathrm{~nm}$ \\
\hline Ge quantum well & $20 \mathrm{~nm}$ \\
\hline $\mathrm{Si}_{0.2} \mathrm{Ge}_{0.8}$ spacer & $100 \mathrm{~nm}$ \\
\hline $\mathrm{Si}_{0.2} \mathrm{Ge}_{0.8}$ buffer & \\
\hline relaxed Ge & $2 \mu \mathrm{m}$ \\
\hline Si (001) substrate & \\
\hline
\end{tabular}


Figure 2

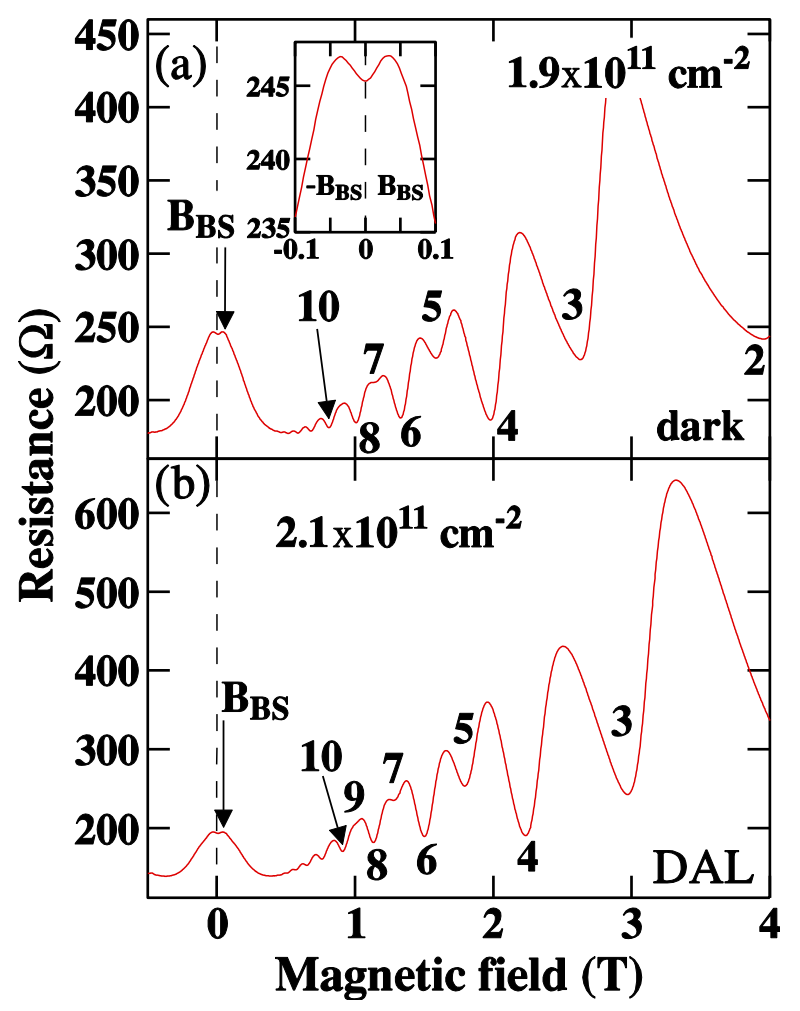


Figure 3

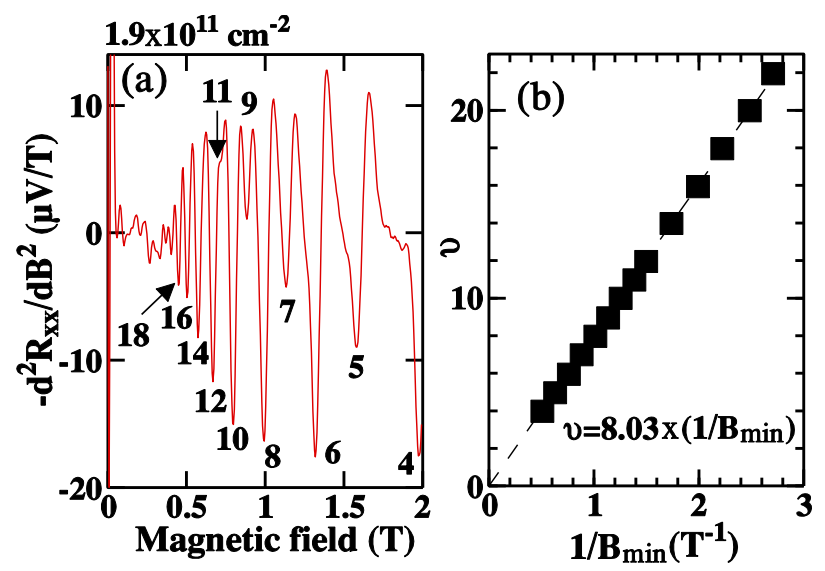


Figure 4
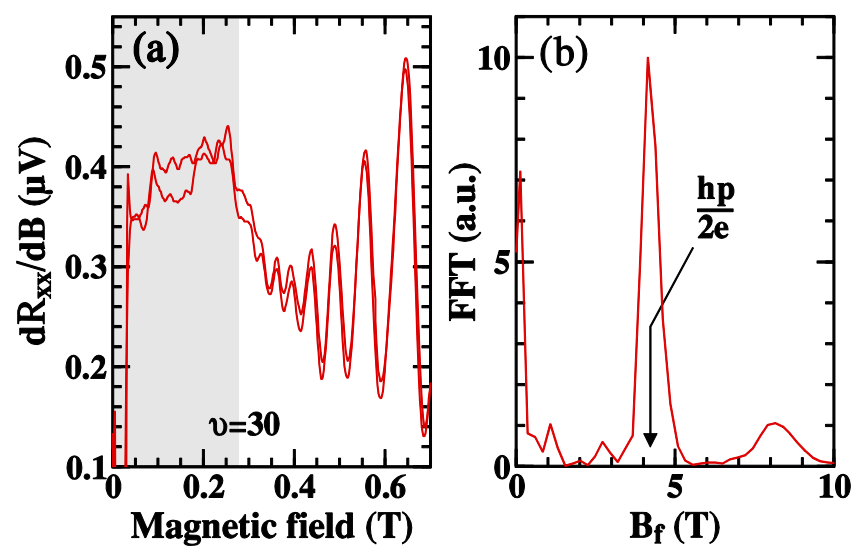
Figure 5

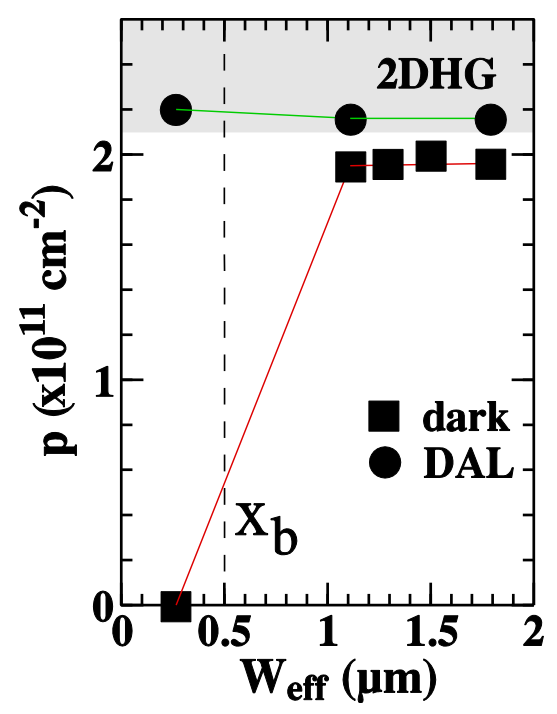

